\title{
New simple composite algorithm for solving multidimensional two-fluid plasma equations
}

\author{
Benoit Morel, Remo Giust, Kazem Ardaneh, Remi Meyer, Francois Courvoisier \\ Institut FEMTO-ST, Université Bourgogne Franche-Comté, CNRS, 15B Avenue des Montboucons, 25030 Besançon cedex, France
}

We developed a new numerical method to build a multidimensional hydrodynamic code solving the complex twofluid plasma model equations. The major advantage of this method is its strong simplicity of implementation but still preserving accuracy and robustness. This approach can be very helpful in the field of laser-plasma interaction, high-intensity optics and plasmonics.

Since the early 1960s the interaction of laser light with a plasma has revealed to be an extremely rich topic in physics and photonics: nonlinear plasmonics, laser-plasma accelerators, inertial fusion [1]. However, the fundamental understanding of the wide range of physical phenomena requires efficient and precise numerical simulations. Dense collisional plasmas, with close-to- or over- critical plasma density are well described by hydro-codes. A hydrodynamic code solves the two-fluid plasma model [2], which means solving Maxwell's equations together with Euler equations describing the plasma dynamics, modeled as a two fluids (mean velocity, local temperature, etc). However, accurate and stable hydro-codes are usually based on Riemann solvers [3], which are extremely complex and out of reach for most non-specialist groups.

Here we report on the development of a new technique to solve this difficult problem for 2D and 3D hydrocodes. Our approach is based on a composite scheme mixing Lax-Friedrichs and Lax-Wendroff schemes to solve Euler equations, and on PseudoSpectral Time-Domain (PSTD) method to solve Maxwell's curl equations. The technical implementation of this approach is simple because it does not require complex mathematical techniques other than finite differences.
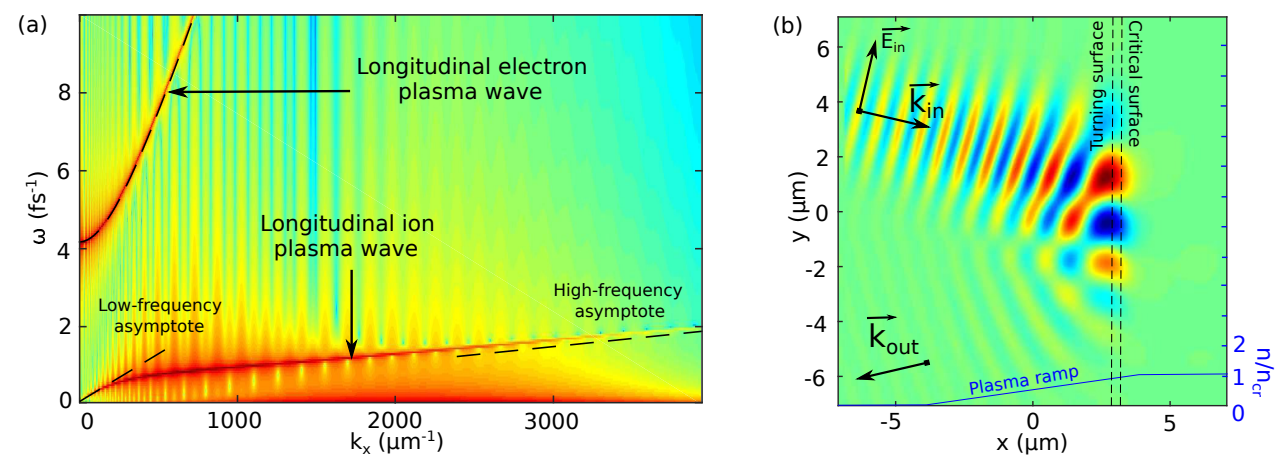

Fig. 1 (a) Logarithm scale spatio-spectral Fourier representation of the excitation of longitudinal waves in a warm plasma with light ions $\left(m_{\text {ion }}=25 m_{\text {electron }}\right)$. Dashed lines show the analytical representations of the dispersion relations for longitudinal electron waves and for the asymptotic values for the dispersion of the ionic longitudinal waves. (b) Spatial distribution of $E_{y}$ laser field component during reflection on over-critical plasma density ramp in oblique incidence.

We validated our code against numerous difficult problems. The composite scheme for solving Euler equations was first validated with the Noh shock test problem [4], known as one of the most demanding shock tests, and with the Rayleigh-Taylor instability problem [5]. Together with PSTD, the full code was then used to reproduce most of the well-known plasma physics problems: propagation in sub-critical plasmas, reflection on over-critical plasmas, inhomogeneous plasmas with linear density profiles and oblique incidence. Fig. 1(a) shows the simulation result of the excitation of longitudinal waves plotted in spectral representation, where the comparison between the analytic and numerical results shows an excellent agreement, even for the ion plasma waves where analytics describe the low- and high-frequency asymptotic regimes. Fig. 1(b) shows another example of test with oblique incidence on over-critical plasma density ramp, in full agreement with the theoretical description.

To conclude, we developed a hydrodynamic code which is very simple to implement. We do not claim that this algorithm is the most accurate or faster than state of the art, but this is a simple and yet very robust way to achieve simulations in the fields of laser-plasma interaction and nonlinear plasmonics.

This research has received funding from H2020 European Research Council (ERC) under grant agreement 682032-PULSAR.

\section{References}

[1] M. Kauranen A.V. Zayats, "Nonlinear plasmonics”, Nat. Photonics 6, 737-748 (2012).

[2] P. Gibbon, Short pulse laser interactions with matter, (Imperial College Press, London, 2005).

[3] U. Shumlak and J. Loverich, "Approximate Riemann solver for the two-fluid plasma model”, J. Comput. Phys. 187, 620-638 (2003).

[4] W.F. Noh, "Errors for calculations of strong shocks using an artificial viscosity and an artificial heat flux", J. Comput. Phys. 72, 78 (1987).

[5] D.L. Youngs, "Numerical simulation of turbulent mixing by Rayleigh-Taylor instability", J. Sci. Comput. 12, 32-44 (1984). 